Portland State University

PDXScholar

Mechanical and Materials Engineering Faculty

Publications and Presentations

$1-1-2010$

\title{
Development of a Contingency Capillary Wastewater Management Device
}

\author{
Evan A. Thomas \\ Portland State University
}

Follow this and additional works at: https://pdxscholar.library.pdx.edu/mengin_fac

Part of the Systems Engineering Commons

Let us know how access to this document benefits you.

\section{Citation Details}

Thomas, E., Development of a Contingency Capillary Wastewater Management Device, International Conference on Environmental Systems 2010

This Article is brought to you for free and open access. It has been accepted for inclusion in Mechanical and Materials Engineering Faculty Publications and Presentations by an authorized administrator of PDXScholar. Please contact us if we can make this document more accessible: pdxscholar@pdx.edu. 


\title{
Development of a Contingency Capillary Wastewater Management Device
}

\author{
Evan A. Thomas, Ph.D. ${ }^{1}$ \\ NASA Johnson Space Center, Houston, Texas, 77058
}

\begin{abstract}
The personal body-attached liquid liquidator (PBALL) is conceived as a passive, capillarydriven, contingency wastewater disposal device. In this contingency scenario, the airflow system on the NASA crew exploration vehicle is assumed to have failed, leaving only passive hardware and vacuum vent to dispose of wastewater. To meet these needs, the PBALL was conceived to rely on capillary action and urine wetting design considerations. The PBALL is designed to accommodate a range of wetting conditions from $0^{\circ}<\Theta_{a d v} \sim 90^{\circ}$, be adaptable for both male and female use, collect and retain up to $1 \mathrm{~L}$ of urine, minimize splash-back, and allow continuous draining of the wastewater to vacuum while minimizing cabin air loss. A subscale PBALL test article, which was tested on NASA's reduced-gravity aircraft in April 2010, successfully demonstrated key components of the design.
\end{abstract}

\section{Nomenclature}

$\alpha=$ half-angle of interior corner

$\Delta \rho \quad=$ difference in density between the gas and the liquid

$h=$ height of liquid as measured from the corner vertex

$L=$ length of PBALL test article critical capillary geometries

$P_{\infty} \quad=$ atmospheric pressure

$P_{2} \quad=$ pressure at interface

$R=$ principle interface radius of curvature radius of PBALL test article critical capillary geometries

$\sigma=$ surface tension of the liquid

$\theta=$ contact angle of the liquid with the solid

$\Theta_{a d v} \quad=$ advancing contact angle

$V \quad=$ characteristic velocity of the flow

\section{Introduction}

$\mathrm{N}$

ASA's Orion crew exploration vehicle (CEV) will require efficient use of mass, volume, energy and consumables given the smaller habitable volume and longer-duration missions for which it is planned compared to those of the Space Shuttle Program. One system that will be adapted is the human waste management subsystem. This hardware is responsible for collecting and disposing of human liquid and solid waste, including urine.

The space shuttle and International Space Station (ISS) currently use rotary fan separators for humidity condensate and wastewater separation. The rotary fan separators process high air-to-liquid flow rate ratios by spinning the multiphase flow against a bowl-like surface in which pitot pumps pick up and transfer the denser liquid. The liquid phase is dumped or processed, and the air phase is returned to the cabin. Unfortunately, the rotary fan separators have been shown to be prone to failure and fouling. Tight tolerances and small orifices do not respond well to biofouling or scaling, and, in general, the rotating nature of the equipment increases the likelihood of failure. The separators have failed numerous times,

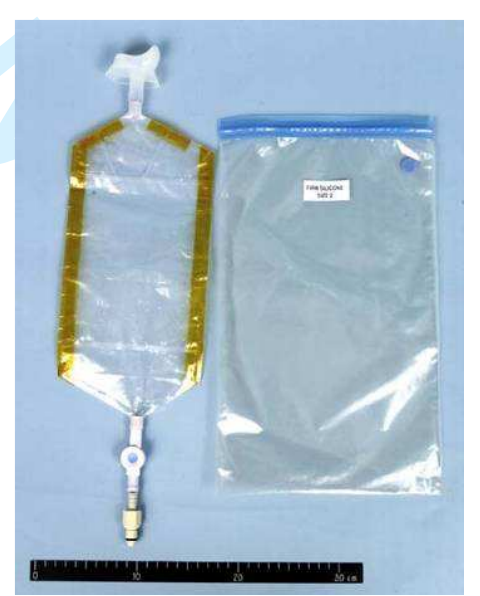

Figure 1. ISS urine collection device.

\footnotetext{
${ }^{1}$ Aerospace Engineer, Crew and Thermal Systems Division, NASA Johnson Space Center, 2101 NASA Parkway, Houston, Texas, 77058 Mail Code EC3AIAA Member.
}

American Institute of Aeronautics and Astronautics 
occasionally putting a severe strain on the mission and the crew. These failures were often linked to wastewater fouling. 1,2

The existing CEV design, which includes a rotary fan separator, also may allow for a contingency wastewater removal device assuming the rotary fan separator has failed. The current baseline contingency device, the ISS urine collection device (UCD), is shown in Fig. 1. However, these UCDs are single-use items that will require significant consumables and are not considered particularly comfortable by female crew members. Instead, this paper presents a proposed capillary-based contingency urine removal system, the personal body-attached liquid liquidator (PBALL), and preliminary subscale testing of this concept.

In the contingency scenario, the airflow system is assumed to have failed, leaving only passive hardware and vacuum vent to dispose of wastewater. To meet these needs, the PBALL was conceived to rely on capillary action and urine wetting design considerations. The PBALL is designed to accommodate a range of wetting conditions from $0^{\circ}<\Theta_{a d v} \sim 90^{\circ}$, be adaptable for both male and female use, collect and retain up to $1 \mathrm{~L}$ of urine, minimize splash-back, and allow continuous drain of the wastewater to vacuum while minimizing cabin air loss.

\section{Background}

\section{A. Microgravity Fluid Management}

Development of spacecraft life support hardware has focused primarily on microgravity applications over the past few decades, with sophisticated designs usually constrained by the limitations of volume, mass, and power. In particular, for two-phase gas/liquid separation in microgravity, centripetal acceleration or capillary action is used to remove liquids without the aid of gravity-driven buoyancy. These systems have often been prone to failure due to fluid fouling caused by biological reactions or mineral scaling, which can clog mechanical systems and degrade the performance of capillary-based systems. In turn, these failures can increase both maintenance cost and the overall crew labor burden.

In a microgravity environment, separation of immiscible fluids (i.e., gas and liquid) does not occur naturally as it does on Earth due to buoyancy. In such situations, surface tension and wetting forces become the dominant influence on liquid behavior. Capillary action is regularly used to control liquids in various spacecraft systems, e.g., propellant and cryogen management, or in thermal fluid loops for temperature control. ${ }^{3}$ Large length-scale capillary systems such as these tend to exploit container geometry and fluid properties to passively transport fluids to desired positions for a variety of purposes. Thus, the shape of the container can serve as a pumping mechanism that does not rely on moving parts. Unfortunately, such methods have only been confidently established for well-characterized systems with favorable wetting conditions. Generally, these systems work best when the advancing contact angle $\Theta_{a d v}$ is reliably low (Fig. 2), yielding a high degree of capillary pumping. This key design parameter, which is the angle formed between the advancing liquid interface and the solid surface as it moves along, is governed by the various surface energies and the geometry of the solid surface. ${ }^{4}$ The lower $\Theta_{a d v}$, the greater the degree of affinity the liquid has for the surface, leading to an increased spontaneous wicking potential. Any changes to surface properties, including fouling, can affect this process.

Systems in microgravity, such as propellant tanks, are often designed to take advantage of capillary forces and passively drive fluids in desired directions. For example, Fig. 3 shows a sketch of a liquid in a wedge in which the pressure drop across the fluid surface can drive the fluid along the corner. The pressure drop can be represented as: ${ }^{5}$

$$
P_{\infty}-P_{2}=\frac{\sigma}{R}=\frac{\sigma}{h}\left(\frac{\cos \theta}{\sin \alpha}-1\right)
$$

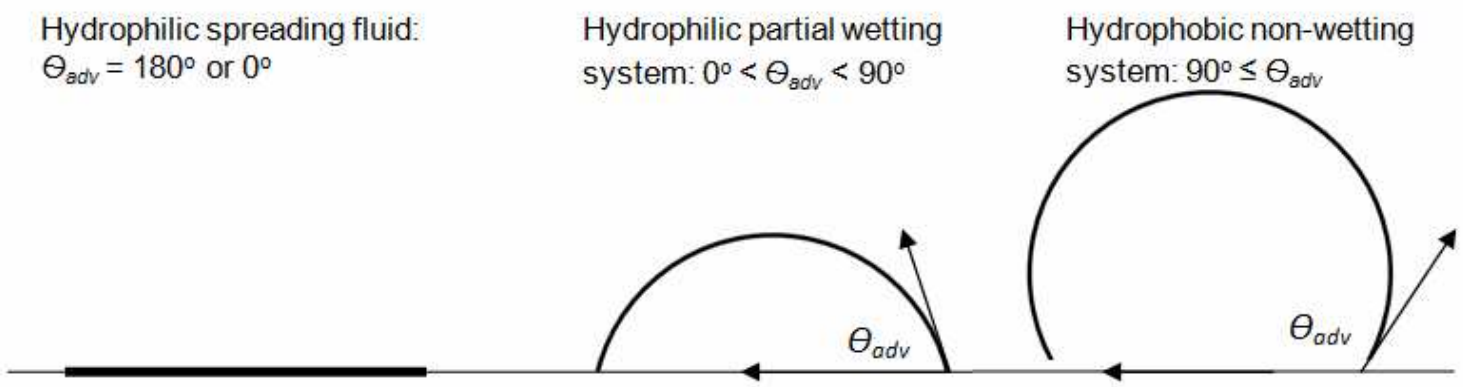

Figure 2. Illustration of advancing contact angle $\left(\theta_{a d v}\right)$ (adapted from Ref. 1).

American Institute of Aeronautics and Astronautics 
where $P_{\infty}$ is the atmospheric pressure, $P_{2}$ is the pressure at the interface, $\sigma$ is the surface tension of the liquid, $R$ is the principle interface radius of curvature, $\theta$ is the contact angle of the liquid with the solid, $\alpha$ is the half-angle of the interior corner, and $h$ is the height of the liquid as measured from the corner vertex. In Eq. (1) when $\alpha<90^{\circ}-\theta$ (and in the absence of significant body forces such as gravity, vehicle acceleration, vibrations, or inertia), an under-pressure $\left(P_{\infty}>P_{2}\right)$ in the liquid arises such that any gradients in the meniscus height along the corner will result in a spontaneous redistribution of fluid along the corner until such gradients are eliminated. Similarly, a corner channel with a changing $\alpha$ will ensure the liquid will pump along the corner in the direction of decreasing capillary pressure. ${ }^{5}$

In contrast to state-of-the-art mechanical approaches, it is anticipated that passive capillary-driven liquid phase separation is feasible in place of active rotary separators for wastewater applications in which the wetting and fouling characteristics can vary widely. Thomas and Muirhead ${ }^{6}$ observed that certain aspects of wastewater fouling can improve wetting characteristics. They found that although vacuum-drying and large defects tended to increase $\Theta_{a d v}$, crystal growth and biofilm growth actually lowered $\Theta_{a d v}$. They also noted the use of pretreatments generally increased $\Theta_{a d v}$. These trends indicate that promotion of wastewater fouling may be exploited to significantly decrease $\Theta_{a d v}$, thereby improving the performance of capillary-based fluid management systems.

In a related study, ${ }^{3}$ a passive capillary-driven "static phase separator" was developed and tested in a reducedgravity environment to demonstrate successful air/liquid separation under highly variable wetting conditions. When the system was operated within design specifications, it achieved $100 \%$ separation in nearly $100 \%$ of the tests performed, and with fluids of widely varying contact angles. The subscale prototype demonstrated several key design features:

- Centrifugal motive gas flow can be used effectively to force droplet coalescence

- The bulk fluid flow may be controlled by either wicking in the case of favorable wetting $\left(\Theta_{a d v}<<90^{\circ}\right)$ or air drag in the case of poor wetting $\left(\Theta_{a d v} \sim 90^{\circ}\right)$; the same geometry can serve both limits

- Capillary forces act to "contain" the liquid in both cases

- Liquid carryover is minimized by pinning edges in the case of favorable wetting or tortuous paths in the case of poor wetting

When contact angles are large, capillary wicking rates are reduced below input liquid flow rate and the capillary force actually resists fluid motion. In this case, bulk liquid will accumulate locally until liquid momentum overwhelms the capillary force and dynamic pressure is sufficient to direct liquid to its desired storage location. Capillary pinning effects in this region then serve to contain the liquid for further processing. In general, geometry-based design considerations based on this principle can be used to improve the operational reliability of capillary-based microgravity fluid management systems.

\section{B. Fouling}

Biofouling is a phenomenon through which microbial growth in a water solution attaches to wetted surfaces within the system. Due to the self-contained and continuous functionality typical of spacecraft water management systems in particular, the detrimental effects of biofouling pose well-recognized design and operational challenges. Testing conducted at NASA Marshall Space Flight Center indicated that microbiology contributed to corrosion of an aluminum alloy used in spacecraft water recovery systems (WRSs). ${ }^{7}$ The study also concluded that once biofilms form in a WRS, they are "extremely difficult to remove" without employing methods that are destructive to hardware. If iodine is added to a potable water system to prevent microbial growth, it must be removed subsequently due to crew health concerns - a unit operation that further increases system complexity. ${ }^{8}$ Designing systems that are less susceptible to biofouling offer only one potential solution to these concerns.

Another form of wastewater fouling is mineral scaling. Scaling occurs as inorganic precipitates form when certain ion concentrations in solutions exceed their solubility limits and create solid salts. These salts then precipitate onto 
surfaces. Because water management systems are often exposed to fluids with high salt concentrations (i.e., urine), the prevention of scaling must be addressed. Scaling can be avoided by pretreatment or careful design and operation of the water management system.

The use of oxidizing pretreatment chemicals for spacecraft wastewater is intended to prevent urea hydrolysis and subsequent biofilm and precipitate formation that tends to foul and clog hardware (e.g., rotary fan separators, vacuum orifice, or WRSs). In contrast, the absence of pretreatment chemicals likely results in an increased prevalence of biofilm formation and small crystalline growth, which has been shown to significantly lower the contact angle

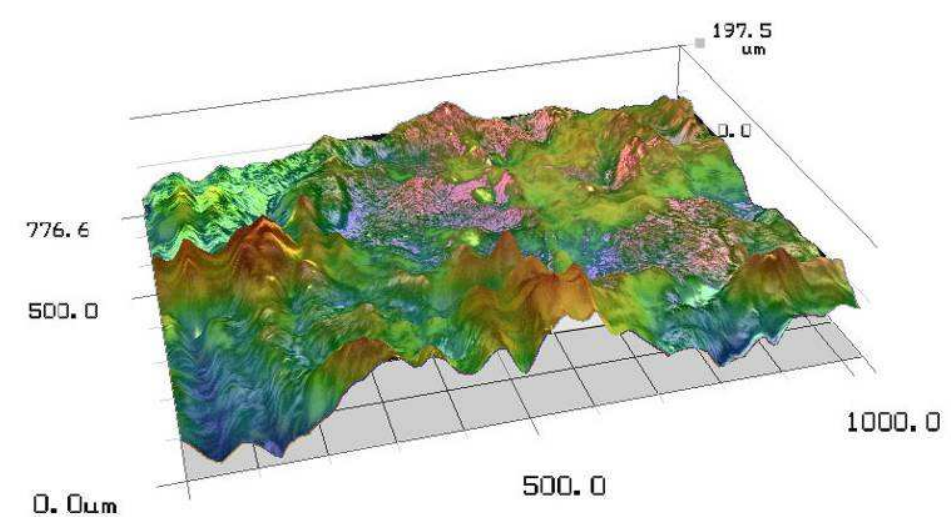

0. Oum

Figure 4. Wastewater fouling profile image. ${ }^{6}$ by up to 44 deg with $95 \%$ confidence. ${ }^{6}$ This response can be used by design to increase performance of a capillarybased wastewater management system. Consequently, an alternate design strategy coinciding with the use of a capillary-based system might be to eliminate the need for pretreatment chemicals intended to prevent fouling, and deliberately allow wastewater fouling to occur in the liquid management system. The operational implications of employing this approach are discussed below.

A wastewater management system that does not require pretreatment chemicals would be beneficial from an operation and logistics perspective, by eliminating hazardous consumables. However, the effect of intentionally allowing biofilm formation on associated systems that are prone to clogging would still need to be considered. As a case study, the space shuttle currently uses a $0.87-\mathrm{cm}$ internal diameter pipeline linking the wastewater storage tank to the vacuum dump orifice, approximately $0.14 \mathrm{~cm}$ in diameter. Between the wastewater tank and the vacuum orifice is a composite foam filter, with the smallest layer having a mean pore size of $300 \mu \mathrm{m}$, yielding a clean filter pressure drop of $0.1 \mathrm{psid}$. During at least one flight anomaly, a decreased flow was observed through the wastewater dump line. A subsequent investigation suggested either bubble formation or precipitate fouling caused the decreased flow rate. ${ }^{9}$ If this conclusion is correct, it is certainly important to consider the likelihood of the intentional fouling layer clogging other parts in the system. Part of this concern arises from the potential for biomass to break off and enter the liquid stream. As the example of a surface with relatively deep features in Fig. 4 illustrates, the thickness of wastewater fouling layers is generally less than $300 \mu \mathrm{m}$, and resulted in minimal formations in the bulk liquid above the test coupon during a 21-day test with wastewater flowing over the surface. These results suggest the fouling layer is unlikely to grow to a depth that would slough off and clog downstream hardware during a 21-day mission anticipated for the CEV. ${ }^{6}$

Thus, while clogging of the wastewater dump line and storage tank is unlikely, care should be taken to appropriately size the dump line filter to avoid clogging it or any small downstream component such as a vacuum orifice. The foam filter is specifically designed to filter particulates that arise in spite of pretreatment chemicals, i.e., clothing fibers or hair. Amorphous deposits are likely a consequence of amorphous crystalline or biofilm formations and must be collected by the filter. It seems feasible to modify the foam filter to include a lower-density stage to trap and remove sticky amorphous deposits without causing a dramatic increase in pressure loss across the filter or allowing breakthrough of the contaminants.

The space shuttle air return line includes both an odor and a bacterial filter. The airflow returned from a capillarybased liquid-air separator would likely have a higher odor and bacterial burden, but could likewise be filtered before returning to the cabin. Of greater concern is the probable odor and bacteria emitted from a wastewater input funnel caused by biofouling in the absence of pretreatment. Simple operational rules could be put in place to minimize these undesirable effects as well, however. For example, simply capping the interface when it is not in use would trap odors in a manner similar to how the Apollo crew capsule urine dump line was used. When operated, the air fan assist would draw cabin air through the system and minimize the potential for contaminants to escape. The urine funnel would also be interchangeable and cleaned between uses, as current operational protocols specify. Within these imposed constraints, the absence of pretreatment chemicals should not create an added odor and bacterial burden in the spacecraft. 


\section{Test Design}

The PBALL concept was tested on NASA's 727 reduced-gravity aircraft with a subscale test article. In such a dynamic reduced-acceleration environment, glevels are typically on the order of 0.1 to $0.001 g_{o}$. Therefore, the critical Bond number must be met to ensure that background accelerations do not destabilize the liquid system. ${ }^{3}$ The Bond number is a dimensionless comparison of the relative magnitude of gravitational and capillary forces. When $B o \gg>1$ gravitational forces dominate. When $B o<<1$, capillary forces dominate. ${ }^{12}$ Adapting the Bond number for the PBALL design:

$$
B o_{c r}=\frac{\Delta \rho g R L}{\sigma}<1
$$

where $\Delta \rho$ is the difference in the density between the gas and liquid, and $R$ and $L$ are the radius and length of the PBALL test article critical capillary geometries. ${ }^{3}$ In a similar way, a critical Weber number condition must also be met to reduce the confounding effects of inertia:

$$
W e_{c r}=\frac{\Delta \rho V^{2} R}{\sigma}<3
$$

where $V$ is a characteristic velocity of the flow. An experimental reduced-gravity apparatus was developed that allowed for circulation of a test liquid through a sequence of test articles. All tests were conducted with deionized (DI) water as an ersatz for wastewater with contact angle $\theta>80 \mathrm{deg}$. Some test articles also used pure ethanol as an ersatz for urine with $\theta \sim 10 \mathrm{deg}$, which assumes highly wetting conditions that may be present with certain materials or surface characteristics. The subscale flow rates used were approximately $20 \mathrm{ml} / \mathrm{min}$
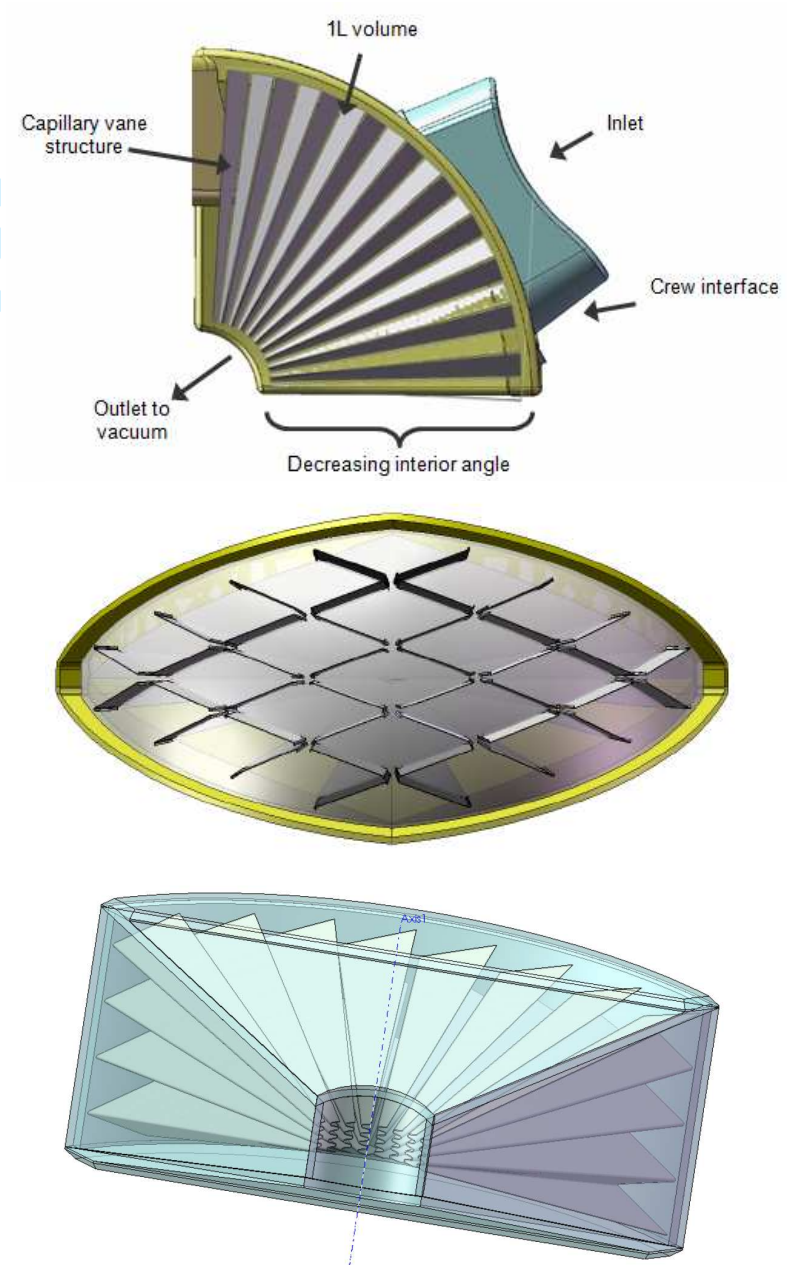

Figure 5. PBALL illustrations. 
liquid flow rate, to allow for examination of capillary phenomenon in the test article during approximately 30 -sec reduced-gravity parabolas, in a test article with a containment volume of approximately $20 \mathrm{ml}$.

A 727 reduced-gravity testing rig was developed to test each SPS subscale test article. The test rig is shown in Fig. 6. The liquid line was fed by graduated syringes, and the liquid drained and carried over to be collected at the end of each test point in separate syringes. The liquid flow path is shown in Fig. 7.

The subscale test article tested on the 727 is shown in Fig. 8. This test article uses a single set of capillary vanes, and liquid is introduced through a nozzle to either the wide or the narrow angles of the vanes, depending on which nozzle input is selected. The vanes are perforated to allow for liquid cross-talk. The test article is constructed entirely of acrylic to allow for flow visualization.

The reduced-gravity test conditions were as follows:

- Two liquids tested: DI water; 200-proof ethanol

- Two nozzle locations: narrow; wide cell input

- 15 to $25 \mathrm{sec}$ of reduced gravity $(<0.01 \mathrm{~g})$

- Input flow rate: 25 to $30 \mathrm{ml} / \mathrm{min}$

A series of duplicate test articles was also constructed out of ABS plastic to perform a wastewater fouling test examining the impact of fouling on capillary contact angle. A subscale test article identical in design to the

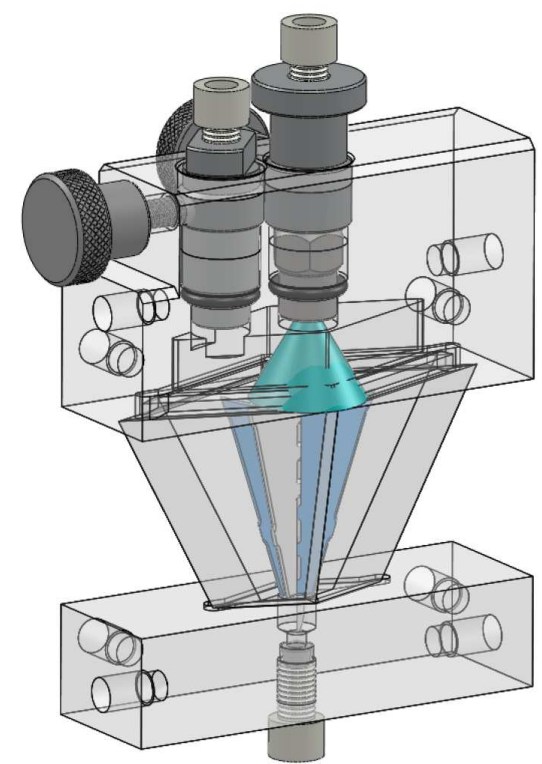

Figure 8. PBALL reduced gravity sub-scale test article optically clear test article used on the 727 was exposed to 23 days of untreated urine, recycling through the system at approximately $160 \mathrm{ml} / \mathrm{min}$. The urine was a single composite batch, which aged over the 23-day test and likely allowed mineral precipitates and befouling to form on the PBALL interior surfaces.

Contact angle measurements were collected on the clean and fouled surfaces in an effort to estimate the appropriate range of contact angles to which the PBALL would reasonably be exposed.

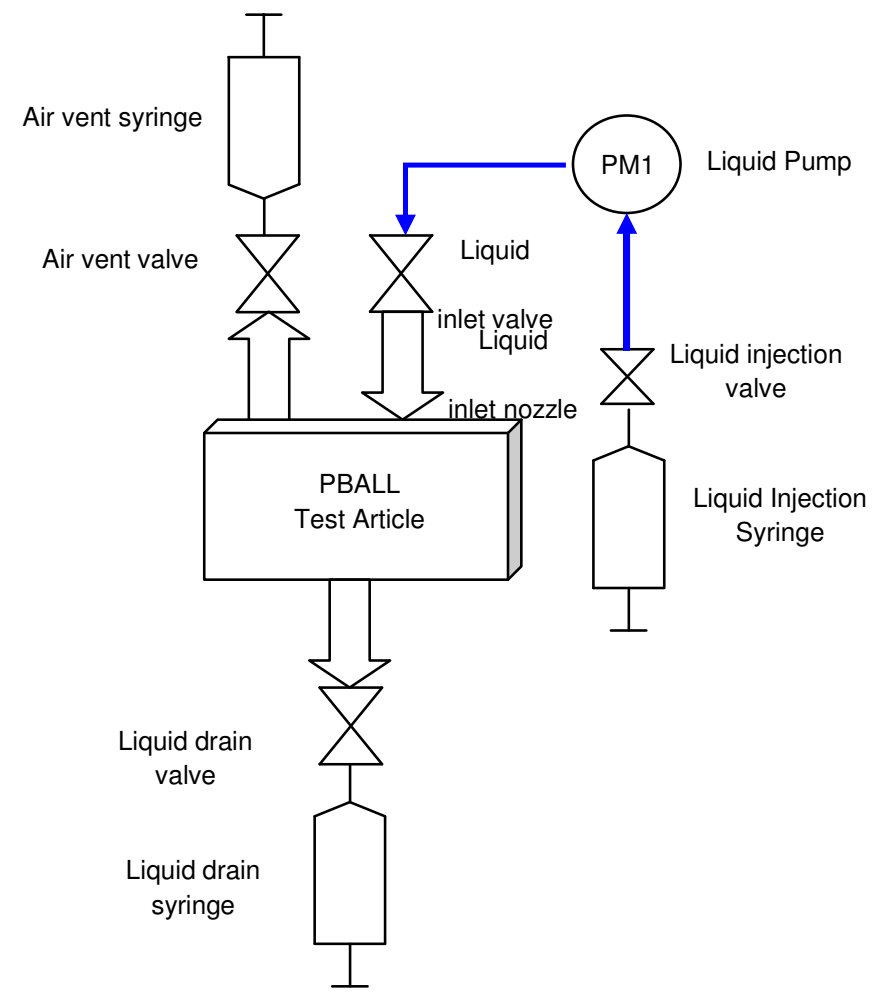

Figure 7. PBALL reduced-gravity testing rig flow loop.

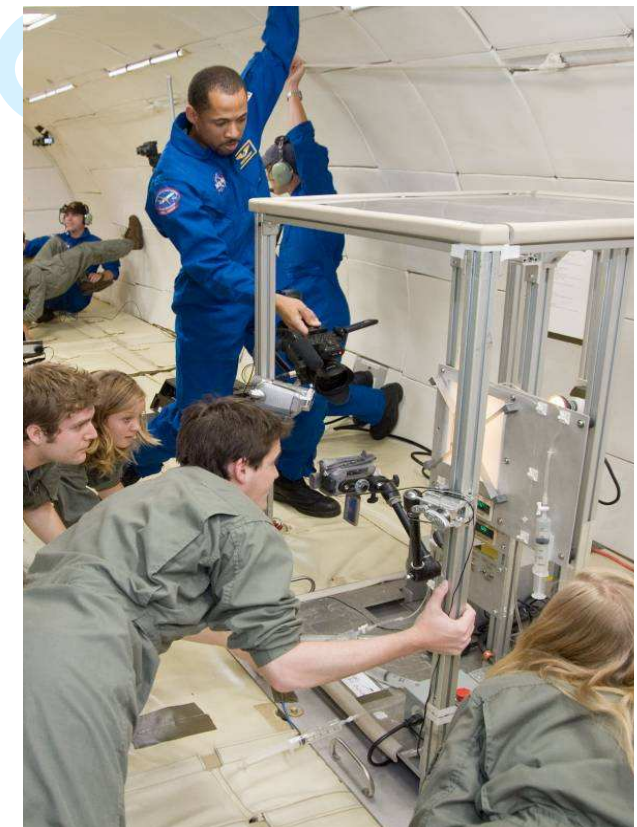

Figure 6. PBALL reduced gravity testing rig 
American Institute of Aeronautics and Astronautics

Representative still-frame images from video collected during the reduced-gravity flights are shown in Fig. 9 and Fig. 10. Figure 9 (left) shows a representative filling event with DI water using the nozzle in the narrow cell. The image also shows a nearly full right-hand cell, with partially filled middle and left-hand cells. Figure 9 (right) shows the same conditions with the nozzle in the wide cell. In these conditions, the cells filled more equally.

Figure 10 shows typical draining events during reduced gravity for DI water (left) and ethanol (right). Generally, the ethanol test points showed improved capillary wetting, as expected, because of the lower advancing contact angle of ethanol compared to DI water. This was demonstrated during both filling and draining events. Internetposted YouTube videos of several test points are available from the author on request.

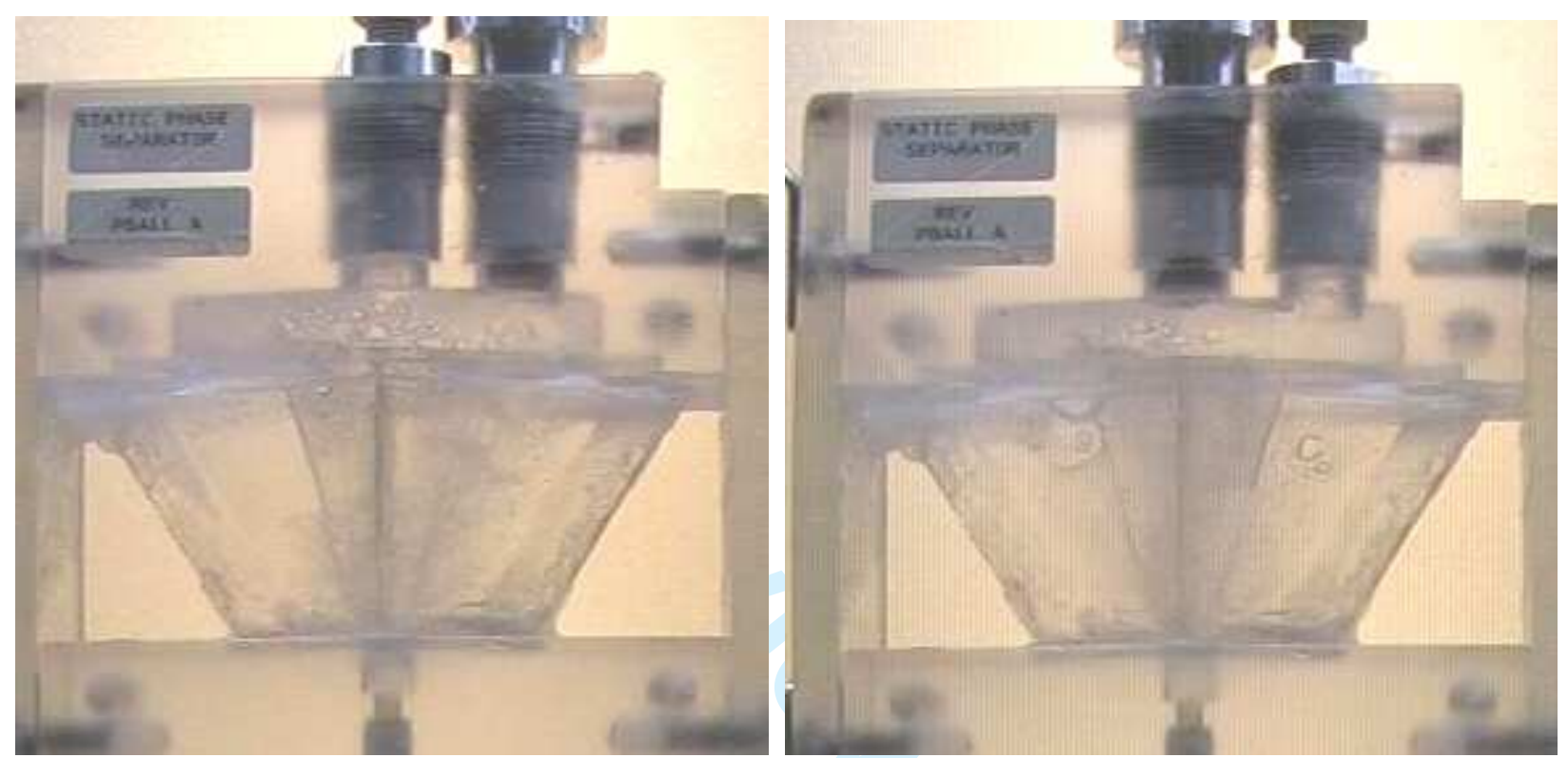

Figure 9. Reduced-gravity filling of DI water at $25 \mathrm{ml} / \mathrm{min}$. Narrow cell nozzle (left); wide cell nozzle (right).
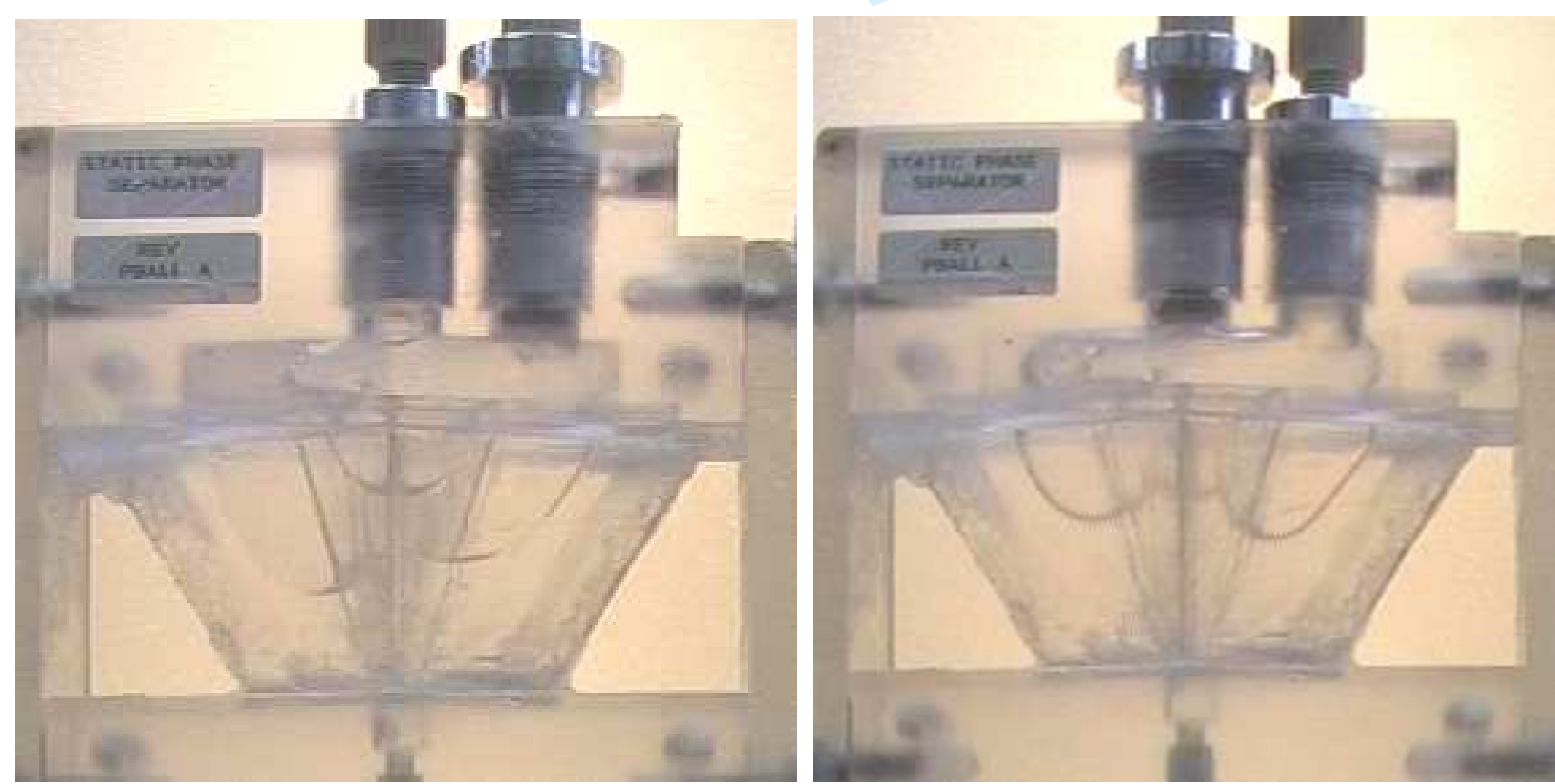

Figure 10. Reduced-gravity draining of DI water (left); filling of ethanol (right). 
Figure 11 shows a plot of 20 reduced gravity test points for the PBALL, showing the injected and drained fluid volumes for each test point, and the running water balance within the PBALL. This plot generally suggests that the PBALL successfully separated the fluid from the gas, was able to drain the fluid as a bulk, and did not retain significant amounts of fluid within the PBALL.

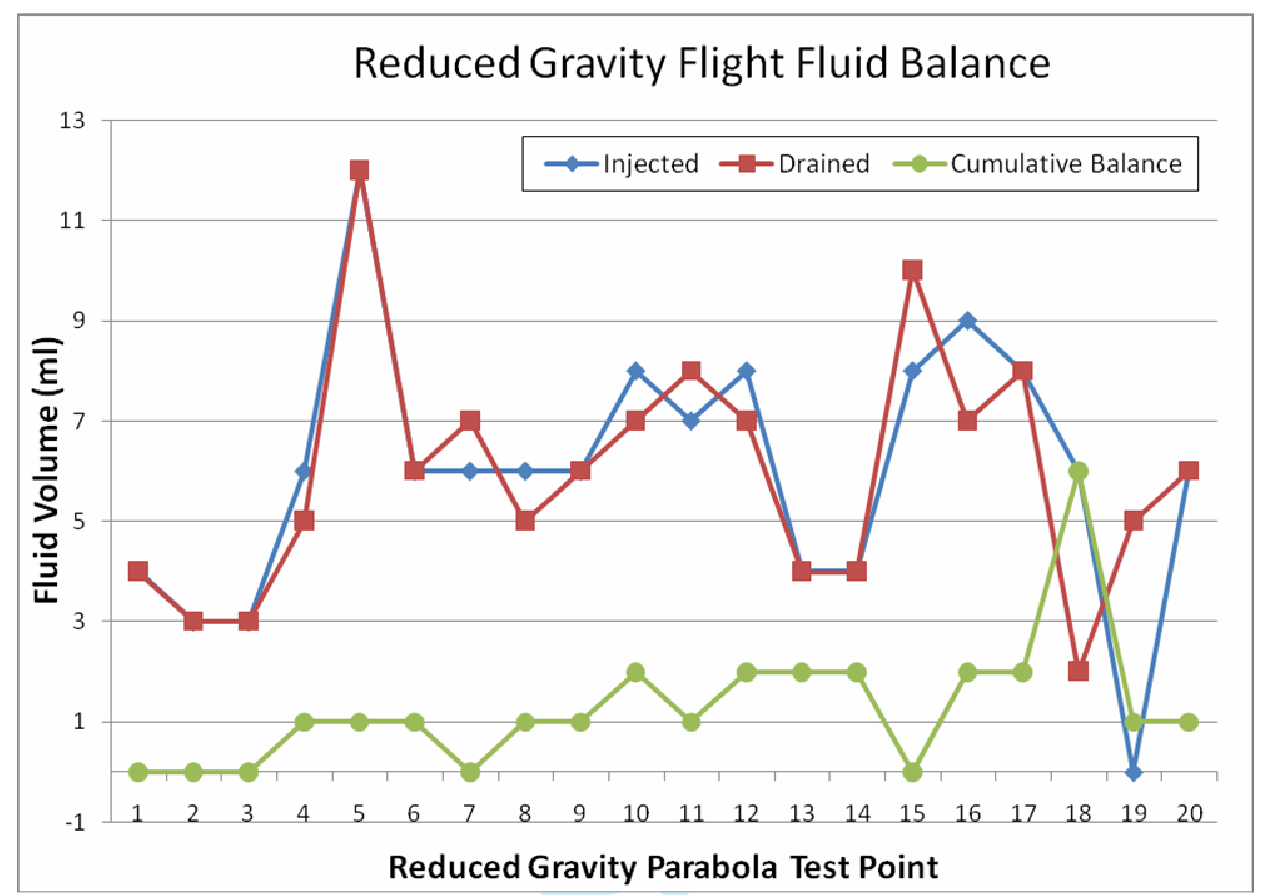

Figure 11. PBALL reduced-gravity flight fluid balance for 20 test points.

While few other quantitative measurments are possible with this type of experiment, significant qualitative observations can be made.

- The test article successfully ccoalesced the spray into a bulk fluid that separated from the air and was contained deep in the cell. This demonstrates the key functional component of the PBALL design: its capability to capture and retain liquid droplets and form a bulk mass away within the cells and separate from the air.

- Both DI water and ethanol generally filled one cell at a time before cross-talking and filling other cells. This phenomona was most pronounced in the narrow cell. When the nozzle introduced spray to the wider cell, filling was more even.

- The system successfully separated both DI water and ethanol, demonstrating its capability to perform in highly variable wetting conditions.

- A pinning edge, serving to partiton cross-talk between cells, was observed at the bottom of the vane structure. This feature is described in more detail below.

Contact line pinning is an effect that is often seen macroscopically due to surface roughness and heterogeneities much smaller than the bulk fluid of interest. These pinning events can result in increased advancing contact angles and contact angle hysteresis. When a pinned system in a metastable state is disturbed, the liquid-solid system assumes the equilibrium contact angle. This is akin to tapping an overfilled glass and spilling water; i.e., this action breaks the hysteresis band at the pinning interface.

The pinning edge in the bottom of the PBALL vane structure was observed when the ethanol was filling the wide cell, drawn down to the bottom of the cell, and then wrapped around the vane to fill adjacent cells. This phenomonon also helps explain the behavior of the liquid filling one cell at a time before capillary pressure overcomes the pinning edge to fill each cell.

This pinning edge is essentially a design defect that can likely be overcome in subsequent design iteratations, which will better ensure equally filling, less-rebound spray as well as better separation and containment of the liquid from the gas.

American Institute of Aeronautics and Astronautics 
Results of the wastewater fouling test are shown in Fig. 12, and representative contact angles are shown in Fig. 13 and Fig. 14. Generally, the urine fouling of the PBALL surfaces served to significantly lower the advancing contact angle by, with 95\% confidence, 41 to $71 \mathrm{deg}$. These results correspond to those presented in Thomas and Muirhead, ${ }^{6}$ wherein biofouling and mineral precipitation induced by wastewater fouling significantly lowered capillary contact angle, thereby improving the performance of capillary-based systems. These results also support reduced-gravity testing results wherein the PBALL was designed to, and successfully, performed when exposed to highly variable wetting conditions such as DI water and ethanol.

\section{Conclusion}

The PBALL concept is conceived as a contingency wastewater removal device for microgravity space flight that relies on capillary geometries to direct liquid inventories without benefit of airflow or rotating equipment.

A subscale test article was demonstrated on NASA's reduced-gravity aircraft, wherein the system successfully coalesced a diffuse spray, separated bulk fluid from the air volume, and drained the bulk fluid in a continuous flow. This was demonstrated for both DI water and ethanol, each of which has highly variable wetting characteristics.

The subscale article also demonstrated an undesired pinning effect at the vane structure base that unintentionally portioned liquid during initial filling events. This design feature may be improved in subsequent iterations to allow more equal filling among cells.

A complementary wastewater fouling study was performed with an identical test article. These results indicated that wastewater fouling of PBALL surfaces serves to lower contact angle and thus improve capillary performance.

Taken together, these results suggest the PBALL design holds promise for being able to separate and retain diffuse liquid input in highly variable wetting conditions.

These results also suggest the continued development of the PBALL design would be worthwhile, including further reduced-gravity flights and, ultimately, a full-scale test article

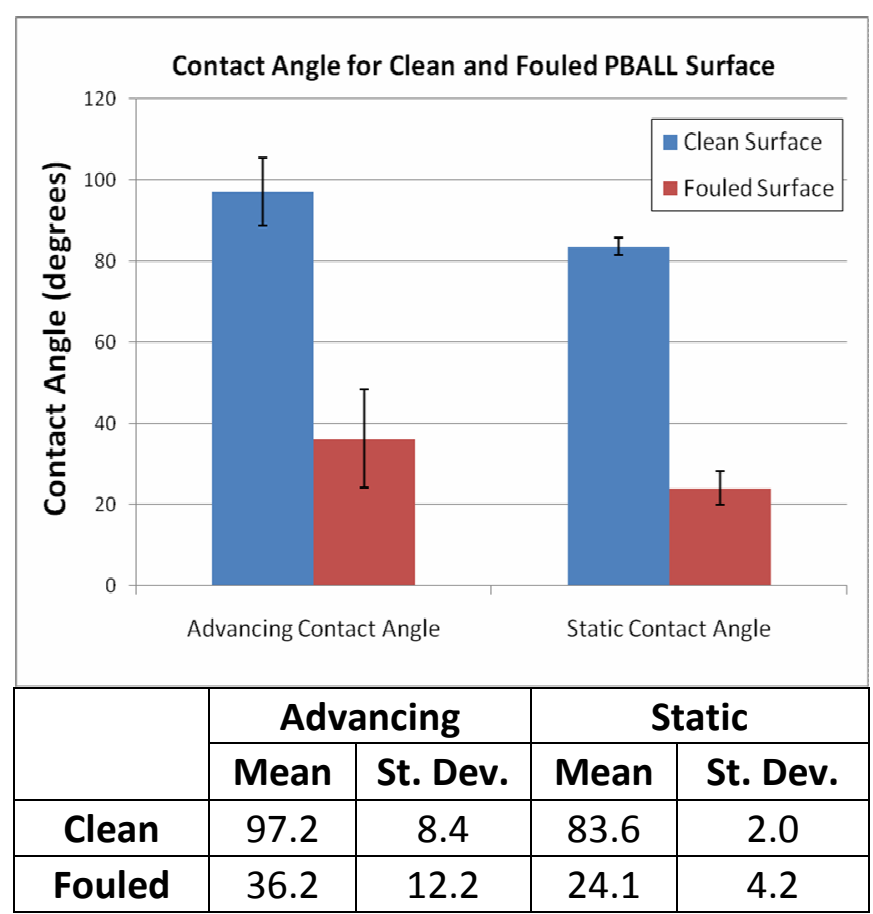

Figure 12. Advancing and static contact angles with standard deviations for clean and wastewater-fouled test article surfaces.

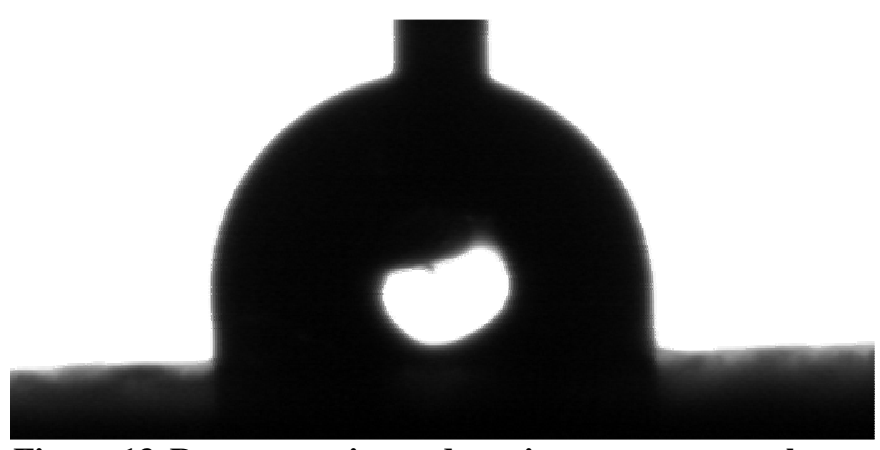

Figure 13. Representative advancing contact angle on clean surface.

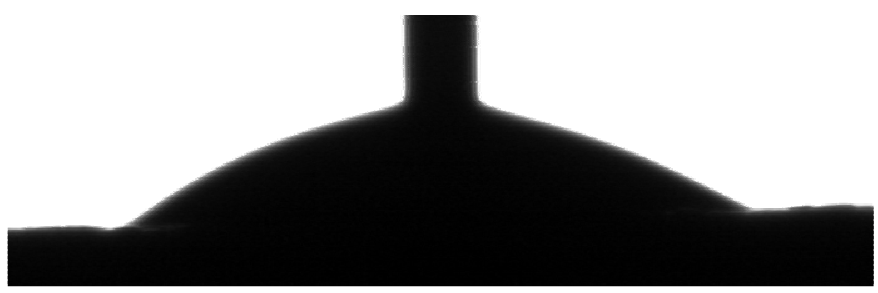

Figure 14. Representative advancing contact angle on fouled surface. experiment on the ISS. In this environment, full liquid volumes and pumping characteristics can be observed. These designs have applicability for myriad fluid-management challenges, including wastewater disposal, water recovery and management, condensate management, and propellant transfer. Systems that demonstrate multiphase fluid management with highly variable wetting conditions without rotary hardware may address critical failure points in spacecraft designs. 


\section{Acknowledgments}

The author is grateful for contributions from Prof. Mark Weislogel, Dr. John Graf, Jeff Stewart, Christie Carlile, Avery Bang, Michael Brackney, Drew Gottula, Jacob Rivera, Jo Goforth, and Douglas Goforth.

\section{References}

${ }^{1}$ Johnson, J., Shuttle OPS V08-Waste Collection System, 2002, NASA-Johnson Space Center: Houston.

${ }^{2}$ Puttkamer, J. ISS On-Orbit Status 05/21/08. International Space Station Daily Report 2008 [cited 2008 November 24, 2008]; Available from: http://www.hq.nasa.gov/osf/iss reports/reports2008/05-21-2008.htm.

${ }^{3}$ Weislogel, M., E. Thomas, and Graf, J., A Novel Device Addressing Design Challenges for Passive Fluid Phase Separations Aboard Spacecraft. Microgravity Science and Technology, 2009. 21(3): p. 257-268.

${ }^{4}$ Quere, D., Wetting and Roughness. Ann Rev. Mater. Res., 2008. 38(23-43): p. 71-99.

${ }^{5}$ Weislogel, M. M., Capillary Flow in an Interior Corner, L.R. Center. 1996, NASA.

${ }^{6}$ Thomas, E., and Muirhead, D., Wastewater Fouling Impact on Capillary Contact Angle. Biofouling, 2009. 2(5): p. 445-454.

${ }^{7}$ Obenhuber, D., Huff, T., and Rodgers, E., Microbial Biofilm Studies of the Environmental Control and Life Support System Water Recovery Test for Space Station Freedom, in ICES1991, SAE: San Francisco, CA.

${ }^{8}$ NASA-JSC, Lunar-Mars Life Support Test Project: Phase III Final Report, 2000, Crew and Thermal Systems Division.

${ }^{9}$ Muirhead, D., and Verostko, C., STS-116 Wastewater Dump Line In-Flight Anomaly Investigation: Experimental Assessment of the STS-116 Wastewater Collection and Effects on Dump Line Subsystem 2007, ESCG.

${ }^{10}$ Thomas, E., Weislogel, M., and Klaus, D., Design Considerations for Sustainable Spacecraft Water Management Systems. Advances in Space Research, 2010. doi: 10.1016/j.asr.2010.04.005.

$\begin{array}{ll}\text { ABS } & \text { acrylonitrile butadiene styrene } \\ \text { CEV } & \text { crew exploration vehicle } \\ \text { DI } & \text { deionized } \\ \text { ISS } & \text { International Space Station } \\ \text { PBALL } & \text { personal body-attached liquid liquidator } \\ \text { UCD } & \text { urine collection device } \\ \text { WRS } & \text { Water Recovery System }\end{array}$

10

American Institute of Aeronautics and Astronautics

http://mc.manuscriptcentral.com/aiaa-ices10 